\title{
Nonuniformly hyperbolic cocycles: admissibility and robustness
}

\author{
LUIS BARREIRA AND CLAUDIA VALLS
}

\begin{abstract}
We give a relatively short proof of the robustness of nonuniformly hyperbolic cocycles in a Banach space under sufficiently small perturbations. In strong contrast to former proofs, we do not need to construct projections leading to the stable and unstable subspaces. Instead, these are obtained fairly explicitly depending only on the boundedness respectively of forward and backward orbits. A difficulty is that we need to construct from the beginning appropriate sequences of Lyapunov norms, with respect to which one can measure the boundedness of the orbits. These norms need not only to be guessed a priori but also all the computations would change if these were not appropriate, both for the original and for the perturbed cocycles. The proof of the robustness is based on the relation between the notions of nonuniform exponential dichotomy and of admissibility, together with nontrivial norm bounds for the expansion and contraction and for the norms of the projections. This relation allows us to construct an invertible operator from the set of bounded perturbations to the set of bounded solutions, and thus to conclude that under sufficiently small perturbations a similar operator exists for the perturbed cocycle.
\end{abstract}

Mathematics Subject Classification (2010): 34D09 (primary); 37D25 (secondary).

\section{Introduction}

Our main aim is to give a relatively short proof of the robustness of nonuniformly hyperbolic cocycles in a Banach space under sufficiently small perturbations. This means that a sufficiently small perturbation of a nonuniform exponential dichotomy is again a nonuniform exponential dichotomy.

A principal motivation for weakening the notion of uniform exponential behavior is given by ergodic theory and the theory of nonuniform hyperbolicity. Namely, consider a flow $\left(\phi_{t}\right)_{t \in \mathbb{R}}$ defined by an autonomous equation $x^{\prime}=f(x)$ in $\mathbb{R}^{n}$ preserving a finite measure $\mu$. This means that

$$
\mu\left(\phi_{t} A\right)=\mu(A)
$$

Partially supported by FCT through CAMGSD, Lisbon.

Received June 27, 2010; accepted February 22, 2011. 
for any measurable set $A \subset \mathbb{R}^{n}$ and any $t \in \mathbb{R}$. One can show that the trajectory of $\mu$-almost every point $x$ with nonzero Lyapunov exponents gives rise to a linear variational equation

$$
v^{\prime}=A_{x}(t) v, \quad \text { with } \quad A_{x}(t)=d_{\phi_{t} x} f,
$$

determining a nonuniform exponential dichotomy. Correspondingly, our results are also a contribution to the theory of nonuniform hyperbolicity. We refer to [1] for a detailed exposition of the theory.

Besides considering the general case of nonuniform hyperbolicity, we also consider the general case when the cocycle is only invertible along the unstable direction. This causes several nontrivial difficulties when compared to former work. In particular, the nonuniform exponential behavior causes that we need to construct from the beginning appropriate sequences of so-called Lyapunov norms, with respect to which the behavior becomes uniform. On the other hand, particularly since there are many Lyapunov norms, one does not know a priori for which ones the actual computations (which must be entirely redone once the norms are changed) actually lead to a proof of the robustness property. Another difficulty is caused by considering cocycles that in general are only invertible along the unstable direction. Namely, after specifying a candidate for unstable subspace of the perturbed cocycle one needs to show that the dynamics is indeed invertible along this subspace.

Due to the central role played by the exponential behavior in a large part of the theory of dynamical systems, it is not surprising that the study of robustness has a long history. In particular, the problem was discussed by Massera and Schäffer [10] (see also [11]), Coppel [6], and in the case of Banach spaces by Dalec'ki $\breve{1}$ and Kreı̆n [7], with different approaches and successive generalizations. For more recent works we refer to $[5,15,17,18]$ and the references therein. We note that all these works consider only the case of uniform exponential dichotomies. We refer to $[2,3]$ for the study of robustness in the more general setting of nonuniform exponential behavior.

Our proof of the robustness is based on the relation between the notions of nonuniform exponential dichotomy and of admissibility, together with nontrivial norm bounds for the expansion and contraction and for the norms of the projections. We recall that the notion of admissibility refers to the existence of bounded solutions for any bounded nonlinear perturbation of the original cocycle. This allows us to construct an invertible operator from the set of bounded perturbations to the set of bounded solutions, and thus to conclude that under sufficiently small perturbations a similar operator exists for the perturbed cocycle. We emphasize that in strong contrast to other works, our main concern is what consequences can be obtained from the admissibility property, and not the admissibility itself, of course independently of its interest in other situations.

Concerning the remaining parts of the proof, as candidates for invariant stable and unstable subspaces we take respectively the forward and backward orbits that are bounded, although now with respect to a sequence of Lyapunov norms, since a posteriori this yields a characterization of these subspaces. This approach is standard in the uniform hyperbolicity theory (for related approaches in the context of 
admissibility see for example $[8,23])$, but now we must address the nonuniform exponential behavior. To estimate the contraction and expansion along the stable and unstable subspaces, we use a similar approach to that in [22] (now for discrete time).

We note that in strong contrast to the proofs of the robustness property presented in [2,3], we do not need to construct projections leading to the stable and unstable subspaces (again, these subspaces are obtained fairly explicitly depending only on the boundedness respectively of the forward and backward orbits).

The study of the admissibility property goes back to pioneering work of Perron in his seminal paper [16], and referred originally to the existence of bounded solutions of the equation

$$
x^{\prime}=A(t) x+f(t)
$$

in $\mathbb{R}^{n}$ for any bounded continuous function $f: \mathbb{R}_{0}^{+} \rightarrow \mathbb{R}^{n}$. This property can be used to deduce the stability or the conditional stability under sufficiently small perturbations of a linear equation. The work of Perron is also a notable contribution to the so-called robustness problem, which asks whether the stability of a linear contraction (or the conditional stability of a linear dichotomy) persists under sufficiently small linear perturbations. In particular, a relatively simple modification of Perron's work yields the following result for discrete time.

Theorem 1.1. Let $\left(A_{m}\right)_{m \in \mathbb{N}}$ be a sequence of $n \times n$ matrices. If for each bounded sequence $\left(f_{m}\right)_{m \in \mathbb{N}} \subset \mathbb{R}^{n}$ there exists $x_{0} \in \mathbb{R}^{n}$ such that the sequence

$$
x_{m}=A_{m-1} x_{m-1}+f_{m}, \quad m \in \mathbb{N}
$$

is bounded, then any bounded sequence $\left(A_{m} \cdots A_{1} x\right)_{m \in \mathbb{N}}$ tends to zero as $m \rightarrow \infty$.

The assumption in Theorem 1.1 is called the admissibility of the pair of spaces in which we take the perturbation $\left(f_{m}\right)_{m \in \mathbb{N}}$ and look for the solution $\left(x_{m}\right)_{m \in \mathbb{N}}$ of relation (1.1). The case when there is simultaneously contraction and expansion is somewhat more complicated (we refer to Section 3 for a detailed discussion).

There is a very extensive literature concerning the relation between admissibility and stability, also in infinite-dimensional spaces. For some of the most relevant early contributions in the area we refer to the books by Massera and Schäffer [11] (culminating the development started with their paper [10]) and by Dalec'kir and Kreı̆n [7]. We also refer to the book [9] for some early results in infinite-dimensional spaces (which are important particularly in view of the applications of the theory to partial differential equations). For more recent work we mention in particular the papers $[8,12-14,19-23]$, and for an additional list of references we refer to the book by Chicone and Latushkin [4] (see in particular the final remarks of Chapters 3 and 4). 


\section{Setup}

Given a sequence $\left(A_{m}\right)_{m \in \mathbb{Z}}$ of linear operators in a Banach space $X$ we define a cocycle by

$$
\mathcal{A}(m, n)= \begin{cases}A_{m-1} \cdots A_{n} & \text { if } m>n, \\ \operatorname{Id} & \text { if } m=n,\end{cases}
$$

for each $m, n \in \mathbb{Z}$. We always assume in the paper that

$$
\left\|A_{n}\right\| \leq c, \quad n \in \mathbb{Z}
$$

for some constant $c \geq 1$. We say that a sequence $\left(A_{m}\right)_{m \in \mathbb{Z}}$ admits a nonuniform exponential dichotomy if there exist:

1. projections $P_{m}$ for each $m \in \mathbb{Z}$ such that

$$
\mathcal{A}(m, n) P_{n}=P_{m} \mathcal{A}(m, n),
$$

with the map

$$
\overline{\mathcal{A}}(m, n):=\mathcal{A}(m, n) \mid \operatorname{ker} P_{n}: \operatorname{ker} P_{n} \rightarrow \operatorname{ker} P_{m}
$$

invertible for each $m \geq n$;

2. constants $D, a>0$ and $\varepsilon \geq 0$ such that

$$
\|\mathcal{B}(m, n)\| \leq D e^{-a(m-n)+\varepsilon|n|}
$$

and

$$
\|\mathrm{C}(n, m)\| \leq D e^{-a(m-n)+\varepsilon|m|}
$$

for every $m \geq n$, with

$$
\mathcal{B}(m, n)=\mathcal{A}(m, n) P_{n} \quad \text { and } \quad \mathcal{C}(n, m)=\overline{\mathcal{A}}(m, n)^{-1} Q_{m},
$$

where $Q_{m}=\mathrm{Id}-P_{m}$ is the complementary projection of $P_{m}$.

Moreover, we say that a sequence $\left(A_{m}\right)_{m \in \mathbb{Z}}$ admits a uniform exponential dichotomy if it admits a nonuniform exponential dichotomy with $\varepsilon=0$.

The following example shows that there exist nonuniform exponential dichotomies that are not uniform, for which (2.1) holds.

Example 2.1. Given $\omega<0$ and $\delta>0$ with $\omega+\delta<0$, we consider constants

$$
A_{m}=e^{\omega+\delta(m+1) \sin \log (m+1)-\delta m \sin \log m+\delta \sin \log (m+1)-\delta \sin \log m}
$$

for $m \in \mathbb{N}$, and $A_{m}=e^{\omega+\delta}$ for $m \in \mathbb{Z} \backslash \mathbb{N}$. We note that

$$
\begin{aligned}
A_{m} & =e^{\omega+2 \delta \sin \log (m+1)-\delta \sin \log m+\delta m[\sin \log (m+1)-\sin \log m]} \\
& \leq e^{\omega+3 \delta+\delta m[\log (m+1)-\log m]}
\end{aligned}
$$


for $m \in \mathbb{N}$. Since

$$
m[\log (m+1)-\log m]=m \log \left(1+\frac{1}{m}\right) \leq m \cdot \frac{1}{m}=1,
$$

we obtain $A_{m} \leq e^{\omega+4 \delta}$ for each $m \in \mathbb{N}$, and thus (2.1) holds. Moreover, for each $m \geq n \geq 1$ we have

$$
\begin{aligned}
\mathcal{A}(m, n) & =e^{\omega(m-n)+\delta m \sin \log (m+1)-\delta n \sin \log n+\delta \sin \log m-\delta \sin \log n} \\
& \leq e^{2 \delta} e^{(\omega+\delta)(m-n)+\delta m(\sin \log m-1)-\delta n(\sin \log n-1)} \\
& \leq e^{2 \delta} e^{(\omega+\delta)(m-n)+2 \delta n} .
\end{aligned}
$$

Since $\omega+\delta<0$, this shows that the sequence $\left(A_{m}\right)_{m \in \mathbb{Z}}$ admits a nonuniform exponential dichotomy with $a=-(\omega+\delta), \varepsilon=2 \delta, D=e^{2 \delta}$, and $P_{n}=$ Id for each $m \in \mathbb{Z}$.

It also follows easily from (2.4) that $\varepsilon$ cannot be taken equal to zero. Indeed, let us write

$$
\mathcal{A}(m, n)=e^{(\omega+\delta)(m-n)+\delta m(\sin \log m-1)-\delta n(\sin \log n-1)+\delta \sin \log m-\delta \sin \log n} .
$$

Given $\eta \in(0,1 / 2)$, we take $m, n \in \mathbb{N}$ with $m \geq n$ such that

$$
|\sin \log m-1|<\eta \quad \text { and } \quad|\sin \log n+1|<\eta .
$$

To see that such integers indeed exist, since the sequence $\log m$ is unbounded, it is sufficient to note that

$$
\log (m+1)-\log m \rightarrow 0 \quad \text { when } \quad m \rightarrow \infty .
$$

Furthermore, the difference $m-n$ can be taken arbitrarily small. We thus obtain

$$
\begin{aligned}
\mathcal{A}(m, n) & \geq e^{(\omega+\delta)(m-n)-\delta m \eta+\delta n(2-\eta)-2 \delta} \\
& =e^{(\omega+\delta-\delta \eta)(m-n)+\delta n(1-\eta)-2 \delta} \\
& \geq e^{(\omega+\delta / 2)(m-n)+\delta n / 2-2 \delta}
\end{aligned}
$$

for each $m \geq n \geq 1$. This shows that $\varepsilon$ cannot be taken smaller than $\delta / 2$. By considering a second component which expands we obtain a nonuniform exponential dichotomy with projections $P_{m}$ different from the identity.

Now let $\left(A_{m}\right)_{m \in \mathbb{Z}}$ be a sequence admitting a nonuniform exponential dichotomy. For each $v \in X$ and $n \in \mathbb{Z}$ we introduce the norm

$$
\|v\|_{n}^{*}=\|v\|_{n}^{s}+\|v\|_{n}^{u}
$$

where

$$
\|v\|_{n}^{s}=\sup \left\{\|\mathcal{B}(k, n) v\| e^{a(k-n)}: k \geq n\right\},
$$

and

$$
\|v\|_{n}^{u}=\sup \left\{\|\mathrm{C}(k, n) v\| e^{-a(k-n+2)}: k \leq n-1\right\} .
$$

It follows readily from (2.2) and (2.3) that the suprema are finite. 
Proposition 2.2. For each $v \in X$ and $n \in \mathbb{Z}$ we have

$$
\frac{1}{c}\|v\| \leq\|v\|_{n}^{*} \leq 2 D e^{\varepsilon|n|}\|v\| .
$$

Proof. By (2.1), for every $n \in \mathbb{Z}$ and $v \in X$ we obtain

$$
\begin{aligned}
\|v\|_{n}^{u}+\|v\|_{n}^{s} & \geq\left\|P_{n} v\right\|+\left\|\overline{\mathcal{A}}(n, n-1)^{-1} Q_{n} v\right\| \\
& \geq\left\|P_{n} v\right\|+\frac{\left\|Q_{n} v\right\|}{\|\overline{\mathcal{A}}(n, n-1)\|} \\
& \geq\left\|P_{n} v\right\|+\frac{1}{c}\left\|Q_{n} v\right\| \\
& \geq \frac{1}{c}\left\|\left(P_{n}+Q_{n}\right) v\right\|=\frac{1}{c}\|v\| .
\end{aligned}
$$

On the other hand, by (2.2) and (2.3) we have

$$
\|v\|_{n}^{s} \leq D e^{\varepsilon|n|}\left\|P_{n} v\right\| \quad \text { and } \quad\|v\|_{n}^{u} \leq D e^{-2 a} e^{\varepsilon|n|}\left\|Q_{n} v\right\| .
$$

This yields the second inequality in (2.5).

\section{Admissibility}

Now we consider the vector space

$$
\mathcal{L}=\left\{(f(n))_{n \in \mathbb{Z}} \subset X: \sup _{n \in \mathbb{Z}}\|f(n)\|_{n}^{*}<\infty\right\},
$$

endowed with the norm

$$
\|f\|^{*}=\sup _{n \in \mathbb{Z}}\|f(n)\|_{n}^{*}
$$

for each sequence $f=(f(n))_{n \in \mathbb{Z}} \subset X$. One can easily verify that $\mathcal{L}$ is a

Banach space. Given $f \in \mathcal{L}$, we define a sequence $x_{f}$ by

$$
x_{f}(n)=\sum_{p=-\infty}^{n} \mathcal{B}(n, p) f(p)-\sum_{p=n+1}^{+\infty} \mathcal{C}(n, p) f(p) .
$$

We want to show that $f \mapsto x_{f}$ is an invertible bounded linear operator with bounded inverse from $\mathcal{L}$ to itself. We start with the following statement.

Theorem 3.1. For each $f \in \mathcal{L}$ :

1. $x_{f}$ is a well-defined sequence in $\mathcal{L}$ with

$$
\left\|x_{f}\right\|^{*} \leq \frac{e^{a}+1}{e^{a}-1}\|f\|^{*}
$$


2. $x_{f}$ is the unique sequence in $\mathcal{L}$ satisfying

$$
x_{f}(n+1)=A_{n} x_{f}(n)+f(n+1), \quad n \in \mathbb{Z} .
$$

Proof. To show that $x_{f}$ is well-defined we first note that

$$
\|\mathcal{B}(n, p)\|^{*}:=\sup _{x \neq 0} \frac{\|\mathcal{B}(n, p) x\|_{n}^{*}}{\|x\|_{p}^{*}} \leq e^{a(p-n)}
$$

for $n \geq p$. Indeed, for each $n \geq p$ we have

$$
\begin{aligned}
\|\mathcal{B}(n, p) x\|_{n}^{*} & =\sup \left\{\|\mathcal{B}(k, n) \mathcal{B}(n, p) x\| e^{a(k-n)}: k \geq n\right\} \\
& =\sup \left\{\|\mathcal{B}(k, p) x\| e^{a(k-n)}: k \geq n\right\} \\
& \leq e^{a(p-n)} \sup \left\{\|\mathcal{B}(k, p) x\| e^{a(k-p)}: k \geq p\right\}=e^{a(p-n)}\|x\|_{p}^{*} .
\end{aligned}
$$

Then

$$
\begin{aligned}
\sum_{p=-\infty}^{n}\|\mathcal{B}(n, p) f(p)\|_{n}^{*} & \leq \sum_{p=-\infty}^{n}\|\mathcal{B}(n, p)\|^{*}\|f(p)\|_{p}^{*} \\
& \leq\|f\|^{*} \sum_{p=-\infty}^{n} e^{a(p-n)}=\frac{1}{1-e^{-a}}\|f\|^{*}
\end{aligned}
$$

Furthermore,

$$
\|\mathcal{C}(n, p)\|^{*}:=\sup _{x \neq 0} \frac{\|\mathcal{C}(n, p) x\|_{n}^{*}}{\|x\|_{p}^{*}} \leq e^{-a(p-n)}
$$

for $n \leq p$. Indeed, for each $n \leq p$ we have

$$
\begin{aligned}
\|\mathcal{C}(n, p) x\|_{n}^{*} & =\sup \left\{\|\mathcal{C}(k, n) \mathcal{C}(n, p) x\| e^{-a(k-n+2)}: k \leq n-1\right\} \\
& =\sup \left\{\|\mathcal{C}(k, p) x\| e^{-a(k-n+2)}: k \leq n-1\right\} \\
& \leq e^{-a(p-n)} \sup \left\{\|\mathcal{B}(k, p) x\| e^{-a(k-p+2)}: k \leq p-1\right\} \\
& =e^{-a(p-n)}\|x\|_{p}^{*} .
\end{aligned}
$$

Then

$$
\begin{aligned}
\sum_{p=n+1}^{+\infty}\|\mathcal{C}(n, p) f(p)\|_{n}^{*} & \leq \sum_{p=n+1}^{\infty}\|\mathcal{C}(n, p)\|^{*}\|f(p)\|_{p}^{*} \\
& \leq\|f\|^{*} \sum_{p=n+1}^{\infty} e^{-a(p-n)}=\frac{1}{e^{a}-1}\|f\|^{*}
\end{aligned}
$$


It follows from (3.4) and (3.6) that

$$
\left\|x_{f}(n)\right\|_{n}^{*} \leq \sum_{p=-\infty}^{n}\|\mathcal{B}(n, p) f(p)\|_{n}^{*}+\sum_{p=n+1}^{+\infty}\|\mathcal{C}(n, p) f(p)\|_{n}^{*} \leq \frac{e^{a}+1}{e^{a}-1}\|f\|^{*},
$$

and in particular the sequence $x_{f}$ is well-defined.

Furthermore,

$$
\begin{aligned}
x_{f}(n+1)= & \sum_{p=-\infty}^{n+1} \mathcal{B}(n+1, p) f(p)-\sum_{p=n+2}^{+\infty} \mathcal{C}(n+1, p) f(p) \\
= & \sum_{p=-\infty}^{n} A_{n} \mathcal{B}(n, p) f(p)+P_{n+1} f(n+1) \\
& +Q_{n+1} f(n+1)-\sum_{p=n+1}^{+\infty} A_{n} \mathcal{C}(n, p) f(p) \\
= & A_{n} x_{f}(n)+f(n+1),
\end{aligned}
$$

and thus $x_{f}$ is a solution of equation (3.2). To show that it is the unique solution in $\mathcal{L}$ we proceed by contradiction. Let $\left(y_{f}(n)\right)_{n \in \mathbb{Z}} \in \mathcal{L}$ be another solution of equation (3.2). Setting

$$
z_{1}(n)=P_{n}\left(y_{f}(n)-x_{f}(n)\right) \quad \text { and } \quad z_{2}(n)=Q_{n}\left(y_{f}(n)-x_{f}(n)\right),
$$

we obtain $z_{1}(m)=\mathcal{B}(m, n) z_{1}(n)$ for each $m \geq n$, and $z_{2}(m)=\mathcal{C}(m, n) z_{2}(n)$ for each $m \leq n$. Therefore, by (3.3), for each $m \in \mathbb{Z}$ and $n \leq m$ we have

$$
\begin{aligned}
\left\|z_{1}(m)\right\|_{m}^{*} & =\left\|\mathcal{B}(m, n) z_{1}(n)\right\|_{n}^{*} \\
& \leq e^{-a(m-n)}\left\|z_{1}(n)\right\|_{n}^{*} \leq e^{-a(m-n)}\left\|z_{1}\right\|^{*} .
\end{aligned}
$$

Letting $n \rightarrow-\infty$ we obtain $\left\|z_{1}(m)\right\|_{m}^{*}=0$, and hence $z_{1}(m)=0$ for all $m \in \mathbb{Z}$.

Similarly, by (3.5), for each $m \in \mathbb{Z}$ and $n \geq m$ we have

$$
\begin{aligned}
\left\|z_{2}(m)\right\|_{m}^{*} & =\left\|\mathfrak{C}(m, n) z_{2}(n)\right\|_{n}^{*} \\
& \leq e^{a(m-n)}\left\|z_{2}(n)\right\|_{n}^{*} \leq e^{a(m-n)}\left\|z_{2}\right\|^{*} .
\end{aligned}
$$

Letting $n \rightarrow+\infty$ we obtain $\left\|z_{2}(m)\right\|_{m}^{*}=0$, and hence $z_{2}(m)=0$ for all $m \in \mathbb{Z}$. This shows that $y_{f}(m)=x_{f}(m)$ for all $m \in \mathbb{Z}$.

The second property in Theorem 3.1 is a version of the so-called admissibility property. The first property allows us to define a linear operator

$$
Q: \mathcal{L} \rightarrow \mathcal{L}, \quad Q(f)=x_{f} .
$$

Moreover, it follows from (3.1) that $Q$ is bounded. 
Proposition 3.2. The operator $Q$ is invertible.

Proof. If $x_{f}=0$, then it follows from (3.2) that

$$
f(n+1)=x_{f}(n+1)-A_{n} x_{f}(n)=0
$$

for every $n \in \mathbb{Z}$, and hence $f=0$. This shows that $Q$ is injective.

Now we show that $Q$ is surjective. Let $g \in \mathcal{L}$. We consider the sequence $f: \mathbb{Z} \rightarrow X$ defined by

$$
f(n)=g(n)-A_{n-1} g(n-1) .
$$

We have

$$
\begin{aligned}
\|f(n)\|_{n}^{*} \leq & \|g(n)\|_{n}^{*}+\left\|A_{n-1} g(n-1)\right\|_{n}^{*} \leq\|g(n)\|_{n}^{*} \\
& +\sup \left\{\left\|\mathcal{B}(k, n) A_{n-1} g(n-1)\right\| e^{a(k-n)}: k \geq n\right\} \\
& +\sup \left\{\left\|\mathcal{C}(k, n) A_{n-1} g(n-1)\right\| e^{-a(k-n+2)}: k \leq n-1\right\} \\
\leq & \|g(n)\|_{n}^{*}+e^{-a} \sup \left\{\|\mathcal{B}(k, n-1) g(n-1)\| e^{a(k-n+1)}: k \geq n\right\} \\
& +\sup \left\{\|\mathcal{C}(k, n-1) g(n-1)\| e^{-a(k-n+2)}: k \leq n-1\right\} \\
\leq & \|g(n)\|_{n}^{*}+e^{-a} \sup \left\{\|\mathcal{B}(k, n-1) g(n-1)\| e^{a(k-n+1)}: k \geq n\right\} \\
& +e^{-a}\left\|Q_{n-1} g(n-1)\right\| \\
& +\sup \left\{\|\mathcal{C}(k, n-1) g(n-1)\| e^{-a(k-n+2)}: k \leq n-2\right\} \\
\leq & \|g(n)\|_{n}^{*}+e^{-a} c\left\|Q_{n-1} g(n-1)\right\|_{n-1}^{*} \\
& +e^{-a} \sup \left\{\|\mathcal{B}(k, n-1) g(n-1)\| e^{a(k-n+1)}: k \geq n-1\right\} \\
& +e^{a} \sup \left\{\|\mathcal{C}(k, n-1) g(n-1)\| e^{-a(k-n+3)}: k \leq n-2\right\} \\
\leq & \|g(n)\|_{n}^{*}+e^{-a} c\|g(n-1)\|_{n-1}^{*}+e^{a}\|g(n-1)\|_{n-1}^{*} .
\end{aligned}
$$

Thus, taking the supremum over $n \in \mathbb{Z}$ we obtain

$$
\|f\|^{*} \leq\left(1+e^{-a} c+e^{a}\right)\|g\|^{*}<+\infty,
$$

which shows that $f \in \mathcal{L}$. Moreover, by construction we have $g=Q(f)$, and so the operator $Q$ is surjective.

It follows from the proof of Proposition 3.2 that the inverse $Q^{-1}: \mathcal{L} \rightarrow \mathcal{L}$ is given by

$$
\left(Q^{-1} g\right)(n)=g(n)-A_{n-1} g(n-1), \quad n \in \mathbb{Z} .
$$

Moreover, by (3.8) the operator $Q^{-1}$ is bounded. 


\section{Robustness}

Now we consider linear perturbations of a nonuniform exponential dichotomy defined by a sequence $\left(A_{m}\right)_{m \in \mathbb{Z}}$ of linear operators. Namely, given another sequence $\left(B_{m}\right)_{m \in \mathbb{Z}}$ of linear operators we consider the cocycle

$$
\mathcal{D}(m, n)= \begin{cases}\left(A_{m-1}+B_{m-1}\right) \cdots\left(A_{n}+B_{n}\right) & \text { if } m>n, \\ \operatorname{Id} & \text { if } m=n .\end{cases}
$$

We define a linear operator $R$ in $\mathcal{L}$ by

$$
R(g)(n)=g(n)-\left(A_{n-1}+B_{n-1}\right) g(n-1), \quad n \in \mathbb{Z} .
$$

This should be compared to (3.9). We also set

$$
b=\sup _{n \in \mathbb{Z}}\left(\left\|B_{n}\right\| e^{\varepsilon|n|}\right) .
$$

To establish the robustness property of nonuniform exponential dichotomies we first obtain an auxiliary statement.

Proposition 4.1. Let $\left(A_{m}\right)_{m \in \mathbb{Z}}$ be a sequence of linear operators admitting a nonuniform exponential dichotomy. If

$$
b<\frac{1}{2 D c\|Q\|},
$$

then $R: \mathcal{L} \rightarrow \mathcal{L}$ is an invertible bounded operator.

Proof. We first show that $R(g) \in \mathcal{L}$ for each $g \in \mathcal{L}$. Indeed, in view of (3.9), by (2.5) and (3.7) we have

$$
\begin{aligned}
\|R(g)(n)\|_{n}^{*} \leq & \left\|\left(Q^{-1} g\right)(n)\right\|_{n}^{*}+\left\|B_{n-1} g(n-1)\right\|_{n}^{*} \\
\leq & \|g(n)\|_{n}^{*}+\left(e^{-a} c+e^{a}\right)\|g(n-1)\|_{n-1}^{*} \\
& +2 D e^{\varepsilon|n|}\left\|B_{n-1} g(n-1)\right\| \\
\leq & \|g(n)\|_{n}^{*}+\left(e^{-a} c+e^{a}+2 D b c\right)\|g(n-1)\|_{n-1}^{*},
\end{aligned}
$$

and taking the supremum over $n \in \mathbb{Z}$ we obtain

$$
\|R(g)\|^{*} \leq\left(1+e^{-a} c+e^{a}+2 D b c\right)\|g\|^{*}<+\infty .
$$

In particular, $R$ is bounded. Moreover, since

$$
\left(Q^{-1}-R\right)(g)(n)=B_{n-1} g(n-1),
$$


we have

$$
\left\|\left(Q^{-1}-R\right)(g)(n)\right\|_{n}^{*} \leq\left\|B_{n-1} g(n-1)\right\|_{n}^{*} \leq 2 D b c\|g(n-1)\|_{n-1}^{*} .
$$

This implies that

$$
\|\mathrm{Id}-R Q\| \leq\left\|Q^{-1}-R\right\| \cdot\|Q\| \leq 2 D b c\|Q\|<1,
$$

and hence $R Q$ is invertible. Since $R=(R Q) Q^{-1}$, we conclude that $R$ is also invertible.

By (3.1) we have $\|Q\| \leq\left(e^{a}+1\right) /\left(e^{a}-1\right)$.

We can now establish the main result of the paper. It shows that the sequence $\left(A_{m}+B_{m}\right)_{m \in \mathbb{Z}}$ admits a nonuniform exponential dichotomy provided that the perturbation $\left(B_{m}\right)_{m \in \mathbb{Z}}$ is ufficiently small.

Theorem 4.2. If the sequence $\left(A_{m}\right)_{m \in \mathbb{Z}}$ admits a nonuniform exponential dichotomy and (4.1) holds, then the sequence $\left(A_{m}+B_{m}\right)_{m \in \mathbb{Z}}$ also admits a nonuniform exponential dichotomy.

Proof. We separate the proof of the theorem into several lemmas. We first introduce some notation. For each $(n, x) \in \mathbb{Z} \times X$ we consider the sequence

$$
s_{n, x}: \mathbb{Z} \rightarrow X, \quad s_{n, x}(k)= \begin{cases}\mathcal{D}(k, n) x, & k \geq n, \\ 0, & k<n .\end{cases}
$$

We also consider the set $U_{n, x}$ of all sequences

$$
u_{n, x}: \mathbb{Z} \rightarrow X, \quad u_{n, x}(k)= \begin{cases}0, & k>n \\ x, & k=n \\ x_{k}, & k<n\end{cases}
$$

such that

$$
x_{k}=\left(A_{k-1}+B_{k-1}\right) x_{k-1} \quad \text { for } \quad k \leq n .
$$

For each $n \in \mathbb{Z}$ we set

$$
E_{n}=\left\{x \in X: s_{n, x} \in \mathcal{L}\right\}
$$

and

$$
F_{n}=\left\{x \in X: U_{n, x} \cap \mathcal{L} \neq \varnothing\right\} .
$$

One can easily verify that $E_{n}$ and $F_{n}$ are vector spaces. We will show that these are stable and unstable subspaces for the sequence $\left(A_{m}+B_{m}\right)_{m \in \mathbb{Z}}$. Incidentally, the conditions in (4.4) and (4.5) essentially correspond to assume that respectively the forward and backward orbits (up to a possible nonuniqueness) are bounded (as it must happen with the stable and unstable subspaces). We first establish the invariance of the subspaces $E_{n}$ and $F_{n}$. 
Lemma 4.3. For each $n \in \mathbb{Z}$ we have

$$
\left(A_{n}+B_{n}\right) E_{n}=E_{n+1} \text { and }\left(A_{n}+B_{n}\right) F_{n}=F_{n+1} .
$$

Proof of the lemma. Since

$$
s_{n+1,\left(A_{n}+B_{n}\right) x}= \begin{cases}\mathcal{D}(k, n) x, & k \geq n+1, \\ 0, & k<n+1,\end{cases}
$$

we have

$$
\left\|s_{n, x}\right\|^{*}=\max \left\{\|x\|_{n}^{*},\left\|s_{n+1,\left(A_{n}+B_{n}\right) x}\right\|^{*}\right\} .
$$

This shows that $s_{n, x} \in \mathcal{L}$ if and only if $s_{n+1,\left(A_{n}+B_{n}\right) x} \in \mathcal{L}$, that is, $x \in E_{n}$ if and only if $\left(A_{n}+B_{n}\right) x \in E_{n+1}$. This yields the first identity in (4.6).

For the second identity, we first note that there is a one-to-one correspondence between the sets $U_{n, x}$ and $U_{n+1,\left(A_{n}+B_{n}\right) x}$. Indeed, if $u_{n, x} \in U_{n+1, x}$, then the sequence $f$ defined by

$$
f(m)= \begin{cases}0, & m>n+1, \\ \left(A_{n}+B_{n}\right) x, & m=n+1, \\ u_{n, x}(m), & m \leq n\end{cases}
$$

is in $U_{n+1,\left(A_{n}+B_{n}\right) x}$, and if $u_{n+1,\left(A_{n}+B_{n}\right) x} \in U_{n+1,\left(A_{n}+B_{n}\right) x}$, then the sequence $g$ defined by

$$
g(m)= \begin{cases}0, & m \geq n+1, \\ u_{n+1,\left(A_{n}+B_{n}\right) x}(m), & m \leq n\end{cases}
$$

is in $U_{n+1, x}$. Thus, without danger of confusion of notation, for two sequences $u_{n, x}$ and $u_{n+1,\left(A_{n}+B_{n}\right) x}$ related in this manner we have

$$
\left\|u_{n+1,\left(A_{n}+B_{n}\right) x}\right\|^{*}=\max \left\{\left\|\left(A_{n}+B_{n}\right) x\right\|_{n+1}^{*},\left\|u_{n, x}\right\|^{*}\right\} .
$$

This shows that $u_{n, x} \in \mathcal{L}$ if and only if $u_{n+1,\left(A_{n}+B_{n}\right) x} \in \mathcal{L}$. Again since there is a one-to-one correspondence between $U_{n, x}$ and $U_{n+1,\left(A_{n}+B_{n}\right) x}$, this shows that there is a one-to-one correspondence between $U_{n, x} \cap \mathcal{L}$ and $U_{n+1,\left(A_{n}+B_{n}\right) x} \cap \mathcal{L}$. Therefore, $x \in F_{n}$ if and only if $\left(A_{n}+B_{n}\right) x \in F_{n+1}$. This yields the second identity in (4.6).

Lemma 4.4. For each $n \in \mathbb{Z}$ we have $X=E_{n} \oplus F_{n}$.

Proof of the lemma. Let $x \in E_{n} \cap F_{n}$. We consider the sequence

$$
g: \mathbb{Z} \rightarrow X, \quad g(k)= \begin{cases}s_{n, x}(k), & k \geq n, \\ u_{n, x}(k), & k \leq n,\end{cases}
$$


for some $u_{n, x} \in U_{n, x} \cap \mathcal{L}$. We note that $s_{n, x}(n)=u_{n, x}(n)=x$. Since

$$
\|g\|^{*} \leq\left\|s_{n, x}\right\|^{*}+\left\|u_{n, x}\right\|^{*}<+\infty,
$$

we have $g \in \mathcal{L}$. Moreover, $g$ is a solution of the equation

$$
x_{n+1}=\left(A_{n}+B_{n}\right) x_{n}+f_{n+1}, \quad n \in \mathbb{Z}
$$

with $f=0$, that is, $R(g)=0$. It follows from Proposition 4.1 that $g=0$, and hence $x=0$. This shows that $E_{n} \cap F_{n}=\{0\}$.

To prove that $E_{n}+F_{n}=X$, let $n \in \mathbb{Z}$ and $x \in X$. We consider the sequence $f: \mathbb{Z} \rightarrow X$ defined by

$$
f(m)= \begin{cases}x, & m=n, \\ 0, & \text { otherwise. }\end{cases}
$$

Clearly, $f \in \mathcal{L}$, so there exists a unique $g \in \mathcal{L}$ such that $R(g)=f$. We note that $g(m)=\mathcal{D}(m, n) g(n)$ for all $m \geq n$. Since $g \in \mathcal{L}$, this shows that $s_{n, g(n)} \in \mathcal{L}$, and hence $g(n) \in E_{n}$. Now we consider the sequence

$$
h: \mathbb{Z} \rightarrow X, \quad h(k)= \begin{cases}0, & k>n, \\ x-g(n), & k=n, \\ -g(k), & k<n .\end{cases}
$$

One can easily verify that $h \in U_{n, x-f(n)}$. Since $h \in \mathcal{L}$, this shows that $h(n)=$ $x-g(n) \in F_{n}$. Therefore,

$$
x=g(n)+(x-g(n)) \in E_{n}+F_{n},
$$

which completes the proof of the lemma.

Now we set $K=\|Q\| /(1-2 D b c\|Q\|)$.

Lemma 4.5. For each $n \in \mathbb{Z}, x \in E_{n}$ and $m \geq n$ we have

$$
\|\mathcal{D}(m, n) x\|_{m}^{*} \leq K\|x\|_{n}^{*} .
$$

Proof of the lemma. Let $n \in \mathbb{Z}$ and $x \in E_{n}$. Given $m \geq n$, we consider the sequence $f: \mathbb{Z} \rightarrow X$ defined by

$$
f(k)= \begin{cases}x, & k=n, \\ 0, & \text { otherwise }\end{cases}
$$

and the sequence $g: \mathbb{Z} \rightarrow X$ defined by

$$
g(k)= \begin{cases}\mathcal{D}(k, n) x, & k \geq n, \\ 0, & k<n .\end{cases}
$$


We note that $g=R^{-1}(f)$. Now we recall that by Proposition 4.1 the operator $R^{-1}: \mathcal{L} \rightarrow \mathcal{L}$ is well-defined. We can write

$$
R=[(R Q-\mathrm{Id})+\mathrm{Id}] Q^{-1},
$$

and thus,

$$
R^{-1}=Q \sum_{k=0}^{\infty}(-1)^{k}(R Q-\mathrm{Id})^{k} .
$$

It follows from (4.3) that

$$
\left\|R^{-1}\right\| \leq\|Q\| \sum_{k=0}^{\infty}\|R Q-\mathrm{Id}\|^{k} \leq \frac{\|Q\|}{1-2 D b c\|Q\|}=K .
$$

herefore, by (4.2),

$$
\begin{aligned}
\|\mathcal{D}(m, n) x\|_{m}^{*} & \leq\|g\|^{*}=\left\|R^{-1}(f)\right\|^{*} \\
& \leq\left\|R^{-1}\right\| \cdot\|f\|^{*} \leq K\|x\|_{n}^{*} .
\end{aligned}
$$

The proof of the lemma is complete.

Lemma 4.6. There exist constants $C>0$ and $\lambda>0$ such that for every $n \in \mathbb{Z}$, $x \in E_{n}$ and $m \geq n$ we have

$$
\|\mathcal{D}(m, n) x\|_{m}^{*} \leq C e^{-\lambda(m-n)}\|x\|_{n}^{*} .
$$

Proof of the lemma. Given $n \in \mathbb{Z}, N \in \mathbb{N}$ and $x \in E_{n}$, we define a sequence $f: \mathbb{Z} \rightarrow X$ by

$$
f(m)= \begin{cases}\mathcal{D}(m, n) x, & n \leq m<n+N, \\ 0, & \text { otherwise. }\end{cases}
$$

By Lemma 4.5, for each $m \in \mathbb{Z}$ we have

$$
\|f(m)\|_{m}^{*} \leq\|\mathcal{D}(m, n) x\|_{m}^{*} \leq K\|x\|_{n}^{*},
$$

and thus, $\|f\|^{*} \leq K\|x\|_{n}^{*}$. On the other hand, we have

$$
R^{-1}(f)(m)= \begin{cases}0, & m<n, \\ (m-n+1) \mathcal{D}(m, n) x, & n \leq m<n+N, \\ N \mathcal{D}(m, n) x, & m \geq n+N\end{cases}
$$


Using Lemma 4.5 with $m$ replaced by $n+N, n$ replaced by $l$, and $x$ replaced by $\mathcal{D}(l, n) x$, we obtain

$$
\begin{aligned}
& \frac{N(N+1)}{2}\|\mathcal{D}(n+N, n) x\|_{n+N}^{*} \\
& =\left\|\sum_{l=n}^{n+N-1}(l-n+1) \mathcal{D}(n+N, n) x\right\|_{n+N}^{*} \\
& \leq \sum_{l=n}^{n+N-1}(l-n+1)\|\mathcal{D}(n+N, n) x\|_{n+N}^{*} \\
& =\sum_{l=n}^{n+N-1}(l-n+1)\|\mathcal{D}(n+N, l) \mathcal{D}(l, n) x\|_{n+N}^{*} \\
& \leq K \sum_{l=n}^{n+N-1}(l-n+1)\|\mathcal{D}(l, n) x\|_{l}^{*} \\
& =K \sum_{l=n}^{n+N-1}\left\|R^{-1}(f)(l)\right\|_{l}^{*} \leq K N\left\|R^{-1}(f)\right\|^{*} .
\end{aligned}
$$

It follows from (4.8) and (4.9) that

$$
\frac{N(N+1)}{2}\|\mathcal{D}(n+N, n) x\|_{n+N}^{*} \leq K^{2} N\|f\|^{*} \leq K^{3} N\|x\|_{n}^{*} .
$$

Therefore,

$$
\|\mathcal{D}(n+N, n)\|^{*}=\sup _{x \neq 0} \frac{\|\mathcal{D}(n+N, n) x\|_{n+N}^{*}}{\|x\|_{n}^{*}} \leq \frac{2 K^{3}}{N+1} .
$$

Now we take $N_{0} \in \mathbb{N}$ sufficiently large so that

$$
s:=\frac{2 K^{3}}{N_{0}+1}<1 .
$$

Given $m, n \in \mathbb{Z}$ with $m \geq n$, we set $r=\left[(m-n) / N_{0}\right]$ where [.] denotes the integer part. Then

$$
\mathcal{D}(m, n)=\mathcal{D}\left(m, m+N_{0} r\right) \mathcal{D}\left(n+N_{0} r, n\right),
$$

and by Lemma 4.5 we have

$$
\begin{aligned}
\|\mathcal{D}(m, n)\|^{*} & \leq K\left\|\mathcal{D}\left(n+N_{0} r, n\right)\right\|^{*} \leq K s^{r} \leq K s^{(m-n) / N_{0}-1} \\
& =(K / s) e^{(m-n)\left(1 / N_{0}\right) \log s}=C e^{-\lambda(m-n)},
\end{aligned}
$$

where

$$
C=K / s \quad \text { and } \quad \lambda=-\left(1 / N_{0}\right) \log s>0 .
$$

This completes the proof of the lemma. 
Lemma 4.7. The subspace $E_{n}$ is closed for all $n \in \mathbb{Z}$.

Proof of the lemma. Let $n \in \mathbb{Z}$ and take $\left(x_{j}\right)_{j \in \mathbb{N}} \subset E_{n}$ with $x_{j} \rightarrow x$ when $j \rightarrow \infty$. By Lemma 4.6 we have

$$
\left\|\mathcal{D}(k, n) x_{j}\right\|_{k}^{*} \leq C e^{-\lambda(k-n)}\left\|x_{j}\right\|_{n}^{*} \leq M C e^{-\lambda(k-n)}
$$

for all $k \geq n$ and $j \in \mathbb{N}$. Letting $j \rightarrow \infty$ in (4.11) we obtain

$$
\|\mathcal{D}(k, n) x\|_{k}^{*} \leq C e^{-\lambda(k-n)}\|x\|_{n}^{*}
$$

for all $k \geq n$, and thus,

$$
\sup _{k \in \mathbb{N}}\|\mathcal{D}(k, n) x\|_{k}^{*} \leq C\|x\|_{n}^{*}<+\infty .
$$

This shows that $s_{n, x} \in \mathcal{L}$, and hence $x \in E_{n}$.

Therefore, $E_{n}$ is closed.

Lemma 4.8. For each $n \in \mathbb{Z}, x \in F_{n}$ and $m \geq n$ we have

$$
\|\mathcal{D}(m, n) x\|_{m}^{*} \geq \frac{\|x\|_{n}^{*}}{K} .
$$

Proof of the lemma. Let $n \in \mathbb{Z}$ and $x \in F_{n}$. By (4.5), there exists a sequence $u_{n, x} \in U_{n, x} \cap \mathcal{L}$. Given $m \geq n$, we consider the sequence $f: \mathbb{Z} \rightarrow X$ defined by

$$
f(k)= \begin{cases}-\mathcal{D}(m, n) x, & k=m, \\ 0, & \text { otherwise }\end{cases}
$$

and the sequence $g: \mathbb{Z} \rightarrow X$ defined by

$$
g(k)= \begin{cases}0, & k \geq m, \\ \mathcal{D}(k, n) x, & n \leq k<m, \\ u_{n, x}(k), & k<n\end{cases}
$$

We note that $g=R^{-1}(f)$. Moreover, since $g(n)=x$ we have $\|g\|^{*} \geq\|x\|_{n}^{*}$. Therefore, by (4.2) and (4.8),

$$
\|x\|_{n}^{*} \leq\|g\|^{*}=\left\|R^{-1}(f)\right\|^{*} \leq K\|\mathcal{D}(m, n) x\|_{m}^{*} .
$$

This completes the proof of the lemma.

It follows from Lemma 4.8 that the map $\mathcal{D}(m, n) \mid F_{n}: F_{n} \rightarrow \mathcal{D}(m, n) F_{n}$ is invertible. Moreover, by Lemma 4.3 we have $\mathcal{D}(m, n) F_{n}=F_{m}$, and hence, the map $\mathcal{D}(m, n) \mid F_{n}: F_{n} \rightarrow F_{m}$ is onto and invertible. For simplicity of the notation, we write $\left(\mathcal{D}(m, n) \mid F_{n}\right)^{-1}=\mathcal{D}(n, m)$. 
Lemma 4.9. For every $n \in \mathbb{Z}, x \in F_{n}$ and $m \leq n$ we have

$$
\|\mathcal{D}(m, n) x\|_{m}^{*} \leq C e^{-\lambda(n-m)}\|x\|_{n}^{*},
$$

with $C$ and $\lambda$ as in Lemma 4.6.

Proof of the lemma. Given $n \in \mathbb{Z}, N \in \mathbb{N}$ and $x \in F_{n}$, we define a sequence $f: \mathbb{Z} \rightarrow X$ by

$$
f(m)= \begin{cases}\mathcal{D}(m, n) x, & n-N<m \leq n, \\ 0, & \text { otherwise. }\end{cases}
$$

It follows from Lemma 4.8 that

$$
\|f(m)\|_{m}^{*} \leq\|\mathcal{D}(m, n) x\|_{m}^{*} \leq K\|x\|_{n}^{*}
$$

for every $m \leq n$, and thus,

$$
f \in \mathcal{L} \quad \text { and } \quad\|f\|_{\infty}^{*} \leq K\|x\|_{n}^{*} .
$$

On the other hand,

$$
\begin{aligned}
\frac{N(N+1)}{2}\|\mathcal{D}(n-N, n) x\|_{n-N}^{*} & =\left\|\sum_{l=n-N}^{n-1}(l-n) \mathcal{D}(n-N, n) x\right\|_{n-N}^{*} \\
& \leq \sum_{l=n-N}^{n-1}(l-n)\|\mathcal{D}(n-N, n) x\|_{n-N}^{*} \\
& =\sum_{l=n-N}^{n-1}(l-n)\|\mathcal{D}(n-N, l) \mathcal{D}(l, n) x\|_{n-N}^{*} .
\end{aligned}
$$

Since

$$
R^{-1}(f)(m)= \begin{cases}0, & m \geq n, \\ (m-n) \mathcal{D}(m, n) x, & n-N \leq m<n, \\ -N \mathcal{D}(m, n) x, & m<n-N,\end{cases}
$$

we obtain

$$
\begin{aligned}
\frac{N(N+1)}{2}\|\mathcal{D}(n-N, n) x\|_{n-N}^{*} & \leq K \sum_{l=n-N}^{n-1}(l-n)\|\mathcal{D}(l, n) x\|_{l}^{*} \\
& =K \sum_{l=n-N}^{n-1}\left\|R^{-1}(f)(l)\right\|_{l}^{*} \leq K N\left\|R^{-1}(f)\right\|_{\infty}^{*}
\end{aligned}
$$

Moreover, by (4.8) we have $\left\|R^{-1}(f)\right\|^{*} \leq K\|f\|^{*}$, and hence, by (4.12),

$$
\frac{N(N+1)}{2}\|\mathcal{D}(n-N, n) x\|_{n-N}^{*} \leq K^{2} N\|f\|_{\infty}^{*} \leq K^{3} N\|x\|_{n}^{*} .
$$


Therefore,

$$
\|\mathcal{D}(n-N, n)\|^{*} \leq \frac{2 K^{3}}{N+1} .
$$

Given $m, n \in \mathbb{Z}$ with $m \leq n$, we set $r=\left[(n-m) / N_{0}\right]$ with $N_{0}$ as in (4.10). Then

$$
\mathcal{D}(m, n)=\mathcal{D}\left(m, m-N_{0} r\right) \mathcal{D}\left(n-N_{0} r, n\right),
$$

and hence,

$$
\begin{aligned}
\|\mathcal{D}(m, n)\|^{*} & \leq K\left\|\mathcal{D}\left(n-N_{0} r, n\right)\right\|^{*} \leq K s^{r} \leq K s^{(n-m) / N_{0}-1} \\
& =(K / s) e^{(n-m)\left(1 / N_{0}\right) \log s}=C e^{-\lambda(n-m)} .
\end{aligned}
$$

This completes the proof of the lemma.

Lemma 4.10. The subspace $F_{n}$ is closed for all $n \in \mathbb{Z}$.

Proof of the lemma. Let $n \in \mathbb{Z}$ and $\left(x_{j}\right)_{j \in \mathbb{N}} \subset F_{n}$ with $x_{j} \rightarrow x$ when $j \rightarrow \infty$. Since $x_{j} \in F_{n}$, there exists a (unique) sequence $u_{n, x_{j}} \in U_{n, x_{j}} \cap \mathcal{L}$. Moreover, since $\mathcal{D}(m, n) \mid F_{n}: F_{n} \rightarrow F_{m}$ is onto and invertible, for each $m \geq n$ we have $\mathcal{D}(k, n) x_{j} \in F_{k}$ for all $k \leq n$. Using Lemma 4.9 we obtain

$$
\begin{aligned}
\left\|x_{j}-x_{l}\right\|_{n}^{*} & =\left\|\mathcal{D}(n, k)\left(u_{n, x_{j}}(k)-u_{n, x_{l}}(k)\right)\right\|_{n}^{*} \\
& \geq(1 / C) e^{\lambda(n-k)}\left\|u_{n, x_{j}}(k)-u_{n, x_{l}}(k)\right\|_{k}^{*}
\end{aligned}
$$

for all $n \geq k$ and $j, l \in \mathbb{N}$. This shows that we can define the sequence

$$
f: \mathbb{Z} \rightarrow X, \quad f(k)= \begin{cases}0, & k>n, \\ \lim _{j \rightarrow \infty} u_{n, x_{j}}(k), & k \leq n .\end{cases}
$$

Clearly, $f \in U_{n, x}$. Moreover, since

$$
\left\|u_{n, x_{j}}(k)\right\|_{k}^{*} \leq C e^{-\lambda(n-k)}\left\|\mathcal{D}(n, k) u_{n, x_{j}}(k)\right\|_{n}^{*}=C e^{-\lambda(n-k)}\left\|x_{j}\right\|_{n}^{*}
$$

for all $j \in \mathbb{N}$ and $k \leq n$, we obtain

$$
\|f(k)\|_{k}^{*} \leq C\|x\|_{n}^{*}, \quad k \leq n .
$$

Therefore, $f \in \mathcal{L}$, and we conclude that $x=f(n) \in F_{n}$. This completes the proof of the lemma.

We proceed with the proof of the theorem. It follows from Lemmas 4.4, 4.7 and 4.10 that $E_{n} \oplus F_{n}=X$, with $E_{n}$ and $F_{n}$ closed subspaces of $X$, for each $n \in \mathbb{Z}$. Let $P_{n}$ and $Q_{n}=\mathrm{Id}-P_{n}$ be the projections associated with this decomposition. Then it follows easily from Lemma 4.4 that $\left(A_{n}+B_{n}\right) P_{n}=P_{n+1}\left(A_{n}+B_{n}\right)$ for all 
$n \in \mathbb{Z}$. Moreover, by Lemmas 4.3 and 4.8 the operator $\mathcal{D}(m, k) \mid F_{k}: F_{k} \rightarrow F_{m}$ is invertible.

Furthermore, for $x \in E_{n}$ it follows from Lemma 4.6 that

$$
\|\mathcal{D}(m, n) x\|_{m}^{*} \leq C e^{-\lambda(m-n)}\|x\|_{n}^{*}, \quad m \geq n .
$$

By (2.5) we obtain

$$
\begin{aligned}
\|\mathcal{D}(m, n) x\| & \leq c\|\mathcal{D}(m, n) x\|_{m}^{*} \leq c C e^{-\lambda(m-n)}\|x\|_{n}^{*} \\
& \leq 2 c C D e^{-\lambda(m-n)+\varepsilon|n|}\|x\|
\end{aligned}
$$

for any $m \geq n$. Similarly, for $x \in F_{n}$ it follows from Lemma 4.9 that

$$
\|\mathcal{D}(m, n) x\|_{m}^{*} \leq C e^{-\lambda(n-m)}\|x\|_{n}^{*}, \quad m \leq n,
$$

and proceeding as in (4.13) we get

$$
\begin{aligned}
\|\mathcal{D}(m, n) x\| & \leq c\|\mathcal{D}(m, n) x\|_{m}^{*} \leq c C e^{-\lambda(n-m)}\|x\|_{n}^{*} \\
& \leq 2 c C D e^{-\lambda(n-m)+\varepsilon|n|}\|x\|
\end{aligned}
$$

for any $m \leq n$. Finally, we estimate the norms of the projections $P_{n}$ and $Q_{n}$. Using the notation in the proof of Lemma 4.4, given $x \in X$ we have $P_{n} x=g(n)$, where $g=R^{-1}(f)$ with $f$ given by (4.7). Therefore,

$$
\left\|P_{n}\right\|=\sup _{x \neq 0} \frac{\|g(n)\|}{\|x\|}=\sup _{x \neq 0} \frac{\left\|R^{-1}(f)(n)\right\|}{\|x\|} .
$$

Using (2.5) we obtain

$$
\begin{aligned}
\left\|R^{-1}(f)(n)\right\| & \leq\left\|R^{-1}(f)(n)\right\|_{n}^{*} \leq\left\|R^{-1}(f)\right\|^{*} \leq\left\|R^{-1}\right\| \cdot\|f\|^{*}=\left\|R^{-1}\right\| \cdot\|x\|_{n}^{*} \\
& \leq 2 D e^{\varepsilon|n|}\left\|R^{-1}\right\| \cdot\|x\|,
\end{aligned}
$$

and hence,

$$
\left\|P_{n}\right\| \leq 2 D e^{\varepsilon|n|}\left\|R^{-1}\right\|
$$

Therefore,

$$
\left\|Q_{n}\right\| \leq\left\|\mathrm{Id}-P_{n}\right\| \leq 1+\left\|P_{n}\right\| \leq \max \left\{1,2 D\left\|R^{-1}\right\|\right\} e^{\varepsilon|n|} .
$$

It follows from (4.13) and (4.14) together with (4.15) and (4.16) that the sequence $\left(A_{m}+B_{m}\right)_{m \in \mathbb{Z}}$ admits a nonuniform exponential dichotomy, and the proof of the theorem is complete.

\section{References}

[1] L. BARREIRA and YA. Pesin, "Nonuniform Hyperbolicity", Encyclopedia of Mathematics and Its Application 115, Cambridge University Press, 2007. 
[2] L. BARREIRA and C. VALLS, Robustness of nonuniform exponential dichotomies in Banach spaces, J. Differential Equations 244 (2008), 2407-2447.

[3] L. BARReIRA and C. VAlls, Robustness of general dichotomies, J. Funct. Anal. 257 (2009), 464-484.

[4] C. Chicone and Yu. Latushkin, "Evolution Semigroups in Dynamical Systems and Differential Equations", Mathematical Surveys and Monographs 70, Amer. Math. Soc., 1999.

[5] S.-N. CHOW and H. LEIVA, Existence and roughness of the exponential dichotomy for skew-product semiflow in Banach spaces, J. Differential Equations 120 (1995), 429-477.

[6] W. Coppel, Dichotomies and reducibility, J. Differential Equations 3 (1967), 500-521.

[7] JU. DALEC'KI I and M. KRE YN, "Stability of Solutions of Differential Equations in Banach Space", Translations of Mathematical Monographs 43, Amer. Math. Soc., 1974.

[8] N. HuY, Exponential dichotomy of evolution equations and admissibility of function spaces on a half-line, J. Funct. Anal. 235 (2006), 330-354.

[9] B. LeVitan and V. ZhiKov, "Almost Periodic Functions and Differential Equations", Cambridge University Press, 1982.

[10] J. MASSERA and J. SCHÄFFER, Linear differential equations and functional analysis. I, Ann. of Math. (2) 67 (1958), 517-573.

[11] J. MASSERA and J. SCHÄFFER, "Linear Differential Equations and Function Spaces", Pure and Applied Mathematics 21, Academic Press, 1966.

[12] M. Megan, B. SASU and A. SASU, On nonuniform exponential dichotomy of evolution operators in Banach spaces, Integral Equations Operator Theory 44 (2002), 71-78.

[13] N. V. MINH and N. T. HUY, Characterizations of dichotomies of evolution equations on the half-line, J. Math. Anal. Appl. 261 (2001), 28-44.

[14] N. V. Minh, F. RÄBIGER and R. SCHNAUBELT, Exponential stability, exponential expansiveness, and exponential dichotomy of evolution equations on the half-line, Integral Equations Operator Theory 32 (1998), 332-353.

[15] R. NAULIN and M. PINTO, Admissible perturbations of exponential dichotomy roughness, Nonlinear Anal. 31 (1998), 559-571.

[16] O. Perron, Die Stabilitätsfrage bei Differentialgleichungen, Math. Z. 32 (1930), 703-728.

[17] V. PLISS and G. SELL, Robustness of exponential dichotomies in infinite-dimensional dynamical systems, J. Dynam. Differential Equations 11 (1999), 471-513.

[18] L. POPESCU, Exponential dichotomy roughness on Banach spaces, J. Math. Anal. Appl. 314 (2006), 436-454.

[19] P. PREDA and M. Megan, Nonuniform dichotomy of evolutionary processes in Banach spaces, Bull. Austral. Math. Soc. 27 (1983), 31-52.

[20] P. Preda, A. Pogan and C. Preda, $\left(L^{p}, L^{q}\right)$-admissibility and exponential dichotomy of evolutionary processes on the half-line, Integral Equations Operator Theory 49 (2004), 405-418.

[21] P. Preda, A. Pogan and C. Preda, Schäffer spaces and uniform exponential stability of linear skew-product semiflows, J. Differential Equations 212 (2005), 191-207.

[22] P. Preda, A. PogAn and C. Preda, Schäffer spaces and exponential dichotomy for evolutionary processes, J. Differential Equations 230 (2006), 378-391.

[23] A. SASU, Exponential dichotomy and dichotomy radius for difference equations, J. Math. Anal. Appl. 344 (2008), 906-920.

Dep. Matemática

Instituto Superior Técnico

1049-001 Lisboa, Portugal

barreira@math.ist.utl.pt

cvalls@math.ist.utl.pt 